\title{
Developing students' competencies and abilities in the courses of civilization and culture

\author{
Dr. Mahmoud Fouad
} \\ Dozent an der Deutschabteilung, Pädagogische Fakultät \\ Abstract
}

The research deals with the concept of "teaching in order to develop competencies and abilities as a term in the field of education development in general and university education in particular as a step towards the transformation of education policies from traditional patterns based on providing students with scientific knowledge to develop the personal competencies and skills that accompany students in their careers and transition from control Curricula and teaching methods with cognitive objectives to the curricula of capacity development and competencies.

The importance of civilization and culture in the acquisition of language has been considered as a separate subject with its research methods and teaching methods. This study seeks to reach a consensus on the definition of the substance of civilization and culture.The study also provides a description of all steps to teach the subject of civilization and culture in order to develop the competencies and abilities of students.

In a practical part, the study implements the above mentioned description in the courses of civilization and culture at the Department of German Language at the Faculty of Education, Ain Shams University, in order to address the students' competencies and abilities, enhance their availability and develop other competencies.

\section{Keywords}

Developing students' competencies and abilities, civilization and culture lessons in Egypt

العدد الرابع والعشرون (الجزء الثانن) 2018 


\section{Kompetenzförderung im Landeskundeunterricht}

\section{تنمية قدرات الطلاب في دروس مادة الحضارة والثقافة د.محمود فُؤاد}

\section{مدرس بقسم اللغة الالمانية - كلية التربية - جامعة عين شمس}

يتتاول البحث مفهوم "التدريس بهدف تنمية الكفاءات والقدرات" كمصطلح حاكم في

مجال تطوير التعليم بشكل عام والتعليم الجامعي بشكل خاص كخطوة نحو تحول سياسات التعليم من الانماط التقليدية القائمة على اكساب الطلاب معارف علمية الى تتمية الكفاءات الشخصية والمهارات التى تصاحب الطلاب في حياتهم العملية والتحول من سيطرة مناهج وطرق التدري ذات الاهداف المعرفية الي مناهج تتمية القدرات والكفاءات. لأهمية مادة الحضارة والثقافة في اكتساب اللغة تم اعتبارها مادة منفصلة لها مناهج البحث وطرق التدري الخاصة بها. تسعى هذه الدراسة إلى التوصل إلى إجماع حول تعريف ماهية مادة الحضارة والثقافة والذي يشوبه الكثير من التداخل وعدم الوضوح. كما تتناول الدراسة المواد الخاصة بالحضارة والثقافة في لائحة قسم اللغة الالمانية بكلية التربية جامعة عين شمس للمرحلة الجامعية الاولى بالدراسة والنقد. تقدم الدراسة تصور كامل الخطوات لتدريس مادة الحضارة والثقافة بهدف تتمية كفاءات وقدرات الطلاب وفي هذا الاطار تحدد الدراسة معايير اختيار المادة العلمية لمادة الحضارة والثقافة. وفي جزء عملي تقوم الدراسة بتطبيق التصور السابق ذكره في دروس مادة الحضارة والثقافة بقسم اللغة الالمانية بكلية التربية جامعة عين شمس بهدف مخاطبة كفاءات الطلاب وقدراتهم وتعزيز المتوافر منها وتتمية وتطوير كفاءات اخرى.

\section{الكلمات الدالة}

تتمية قدرات الطلاب، دروس مادة الحضارة والثقافة في مصر 


\section{$\overline{\text { Kompetenzförderung im Landeskundeunterricht }}$ Dr. Mahmoud Fouad Dozent an der Deutschabteilung, Pädagogische Fakultät}

\section{Einleitung}

Der Begriff "kompetenzorientierter Unterricht" ist in der aktuellen, fachdidaktischen Diskussion zum Schlagwort geworden. Dabei erwerben die Lernenden neben spezifischen, sprachlichen Kompetenzen ein angemessenes Handlungswissen, das über die Grenzen des traditionellen FSU hinausgeht und einen wesentlichen Beitrag zum Lernen, aber auch zur Persönlichkeitsentwicklung leistet. Ein Paradigmenwechsel vollzieht sich dementsprechend im Bezug auf die Lehr- und Lernziele. Die Studie möchte hiermit die didaktisch-methodische Diskussion in Ägypten auf der Ebene der Hochschule wie der Schule dazu anregen, Kompetenzmodelle anstatt von kognitiven Ansätze einzusetzen und Kompetenzförderung anstatt von Faktenwissen als Ziel der Ausbildung zu betrachten.

In jeder Form verbaler wie nonverbaler Kommunikation spielt die Landeskunde (LK) eine entscheidende Rolle, indem sie in der Regel kulturspezifische Eigenarten des Ziellandes hervorhebt. Für ihre Relevanz zu einem umsichtigen Spracherwerb betrachtet die Auslandsgermanistik die LK als ein eigenes Fach mit Faktenwissen und Kommunikationstechniken. Ob Lehrbücher separate bzw. klar markierte Anteile oder Module für LK spezialisieren, gilt abgesehen davon jeder authentische Text oder jedes Bild als einen landeskundlichen Hinweis und Beitrag zum Zielkulturwissen.

Diese Studie versucht einen übergreifenden Konsens über die heikle Definition von LK herzustellen und eine wissenschaftlich fundierte Konzeption des Faches in einem kompetenzorientierten DaF-Unterricht zu entwerfen. Es wird sich ferner mit den Kriterien der Auswahl landeskundlichen Stoffes beschäftigt. In einem empirischen Teil unterbreitet die Studie einen praktikablen Vorschlag zum didaktischen Einsatz von LK zur Kompetenzbildung

العدد الرابع والعشرون (الجزء الثانى)

()

مجلة كلية التربية- جامعة عين شمس17 
an der Deutschabteilung der Pädagogischen Fakultät der Universität Ain Shams. Darüber hinaus setzt sich die Studie analytisch mit der Position der LK und deren Lehrplan an derselben Abteilung.

\section{Die Hochschulausbildung, ein Perspektivenwechsel}

Was die PISA-Studien für die Schulreform in Europa bedeuten, ist der Bologna-Prozess für die Hochschulreform, die wesentlich auf "Förderung der Mobilität und arbeitsmarkt- bezogenen Qualifizierung", "lebenslanges Lernen" und "internationale Wettbewerbsfähigkeit" (Bologna 1999: 2f) zielt. Dies ist durch die Bildung der "notwendigen Kompetenzen für die Herausforderungen des neuen Jahrtausends" (ebd.: 1) zu realisieren. Die Universität vermittelt eine breite Wissensgrundlage, muss aber auch durch "Freizügigkeit in der Praxis" (ebd.: 4) auf den Arbeitsmarkt vorbereiten. Der Hochschultradition nach Humboldt'shem Ideal, nämlich Einheit von Forschung und Lehre, sind neue Aufgaben gestellt. Diese sind Kompetenzbildung und Employability. "Generell herrscht eine Verschiebung vom Faktenlehren zum Kompetenzen-Vermitteln, wobei hier die Kompetenz, sich Wissen anzueignen, eine zentrale Rolle eingenommen hat" (Hiller 2011: 5). Die kompetenzorientierten Module werden heutzutage von der Akkreditierungspolitik hochgeschätzt. So entwickelt sich im universitären Kontext eine am „Kompetenzbegriff orientierte Bildungssemantik“ (Otten 2006: 57).

Daher steht der Begriff "Schlüsselkompetenz" bzw. "allgemeine berufsqualifizierende Kompetenzen" seit der Bologna-Reform auf Hochkonjunktur. Learing outcomes, Praxis- und Berufsfeldorientierung sind die leitenden Begriffe dieser Hochschulreform, die die Frage hervorhebt, welche Schlüsselkompetenzen Studierende durch das Studium erwerben sollen. Studierende sollen "dazu befähigt werden, im Studium und im Laufe ihres Arbeitslebens immer wieder flexibel auf unterschiedliche Anforderung zu reagieren, eigenständig Probleme $\mathrm{zu}$ lösen und adäquat mit sachlichen Herausforderungen und Kolleg/innen umzugehen" (Nünning 2008: 2). Für ein erfolgreiches

العدد الرابع والعشرون (الجزء الثانى) 
Studium und einen gelungenen Einstieg in die Karriere soll sich der Lernende erstens seiner Kompetenzen bewusst werden, dann daran arbeiten, sie fortentwickeln und sich durch berufspädagogischdidaktische Orientierung neuere, erforderliche Kompetenzen aneignen.

Die Notwendigkeit zur Schulung von Schlüsselkompetenzen wird durch die Globalisierung gestärkt auch im DaF-Bereich. Der Absolvent soll nicht nur über gute Fremdsprachenkenntnisse verfügen, sondern auch viel wichtiger über andere Kompetenzen. "Interkulturelle Kompetenz wird zu jenen allgemeinen Schlüsselqualifikationen und soft skills gezählt, welche - wie u.a. Führungskompetenz oder Teamfähigkeit - das in einem bestimmten Beruf benötigte Fachwissen und fachliches Können ergänzen" (Straub 2007: 34). Im Vorwort des GERS steht: "Grundlage für die deutschsprachige Fassung der Skalen [...] waren die deutschen Kompetenzbeschreibungen" (Trim 2001: 3), worauf das ganze, fünfte Kapitel der Referenzrahmen "Die Kompetenzen der Sprachverwendenden/Lernenden" eingeht.

\subsection{Schlüsselkompetenzen, eine Begriffsbestimmung}

Schlüsselqualifikationen ermöglichen dem Individuum "sich immer wieder neue Qualifikationen - und damit lebenslang Handlungsfähigkeit in möglichst vielen Bereichen - zu erschließen" (Honolka 2003: 5). Sie sind "die bei Individuen verfügbaren oder durch sie erlernbaren kognitiven Fähigkeiten und Fertigkeiten, um bestimmte Probleme zu lösen, sowie die damit verbundenen motivationalen, volitionalen und sozialen Bereitschaften und Fähigkeiten, um die Problemlösungen in variablen Situationen erfolgreich und verantwortungsvoll nutzen zu können" (Weinert 2003: 27f). Diese Definition verbindet die kognitiven Fähigkeiten mit Fertigkeiten, Wissen mit Können, akademisches Wissen mit Handlungswissen. Die Kompetenzen sollen entdeckt werden und sind gleichzeitig erlernbar. Ein weiterer Zentralaspekt ist die Problemlösungskompetenz. Das Fachwissen ist kein Selbstzweck, sondern ein Weg zur Authentizität, zum konkreten Handeln. Das
العدد الرابع والعشرون (الجزء الثاني) 2018
()
مجلة كلية التربية- جامعة عين شمس 19 
bedeutet, dass erst der verantwortungsvolle Einsatz des Fachwissens wie der operationalen Fertigkeiten zur Kompetenzbildung führt. Neben der Definition hat Nünnig die Merkmale der Schlüsselkompetenzen bestimmt.

\section{Merkmale der Schlüsselkompetenzen:}

a- Sie sind globale, übertragbare Kompetenzen.

b- Sie bezwecken die Qualifikation für vielfältige Funktionen.

c- Sie unterscheiden sich von fachlichen Fähigkeiten und Kenntnissen so, dass sie mit keiner bestimmten Fachdisziplin, sondern mit disziplinübergreifendem Können zu tun haben. Das Fachwissen beschränkt sich auf das erlernte Fach, die Schlüsselkompetenzen eröffnen hingegen wegen ihrer Transferierbarkeit mehrere Berufsperspektiven (vgl. Nünning 2008: 5).

\subsection{Fachliche Kompetenz und Schlüsselkompetenz}

Das Makroziel der Ausbildung ist nicht mehr die Aneignung vom Fachwissen, sondern "die Gewinnung von Grundkompetenzen in möglichst allen Bereichen des Lebens für die kritische Auseinandersetzung mit der gesamten physischen und geistigen Wirklichkeit des Lebens" (Olechowski 1997: 368). Dies kann mithilfe neuer, didaktischer Ansätze umgesetzt. Daher ist der Begriff "Schlüsselqualifikation" vom gegenwärtigen Bildungsdiskurs nicht mehr wegzudenken. "Diese Fähigkeiten, Einstellungen und Haltungen reichen über die fachlichen Fähigkeiten und Kenntnisse hinaus und überdauern sie. Qualifikationsziel ist die berufliche Flexibilität und Mobilität" (Beck 1993: 17f).

Im Unterschied zu den fachlichen Kompetenzen bezieht sich der Begriff "Schlüsselkompetenzen" auf praxisbezogene, soziale und interkulturelle Fähigkeiten. Ein anderer Unterschied wäre: "Anders als bei den fachlichen Qualifikationen ist es nicht üblich, sie im Rahmen eines eigenständigen, formalisierten Verfahrens $\mathrm{zu}$ 


\section{Dr. Mahmoud Fouad}

beurteilen. Sie wirken sich jedoch unmittelbar auf die Prüfungsleistungen aus und beeinflussen deren Bewertung durch die Prüfer" (Drügh 2012: 3). Die Schlüsselkompetenzen entwickeln die Persönlichkeit der Lernenden mit dem Ziel auf Berufs- und Praxisbezogenheit, daher lassen sie sich nicht durch konventionelle Evaluationsverfahren bewerten, doch dadurch sind die Lernenden in der Lage, sich Wissen nicht nur anzueignen, sondern auch es zu verwalten und anzuwenden.

Schlüsselkompetenzen werden je nach der Studium- bzw. Berufsspezifik kategorisiert. Beck (1993), Honolka (2003), Nünning (2008), Drügh (2012) u. a. haben ihre eigene Auflistung von Schlüsselqualifikationen erstellt. Drügh hat die folgenden vier Schlüsselkompetenzen gezählt, die für Studium und Beruf essenziell nötig sind (vgl. Drügh 2012: 3):

A. Text- und Analysekompetenz:

Die Lernenden sind demnach in der Lage, sich ein Bewusstsein für Textsortenstruktur und Thematik der eingeführten Materialien zu verschaffen, die Texte auf ihre Schlüsselinhalte zu rekapitulieren und sie kritisch $\mathrm{zu}$ reflektieren. „Texte sind Lernmittel und Lerngegenstand und auch Lernprodukt" (Riemer 2007: 163).

B. Darstellungs- und Schreibkompetenz:

Die Lernenden erschließen landeskundliche Bezüge aus den eingeführten Materialien induktiv analytisch, paraphrasieren sie schriftlich und stellen sie adressatengerecht dar (vgl. Frank et al 2007: 116ff). Die Optimierung der Schreibkompetenz benötigt andere ergänzende Kompetenzen wie die Formulierungs-, Überarbeitungskompetenz und grammatische wie lexikalische Kompetenz.

C. Moderations- und Präsentationskompetenz:

Die Lernenden haben ihre Textanalyse und Textbearbeitung für andere Kursteilnehmer zu veranschaulichen. Für die Perfektionierung der Präsentation brauchen Lernende Informationsund Medienkompetenz.

D. Informations- und Medienkompetenz: 
Die Lernenden sollen über Techniken der themengerechten Recherche in Bibliotheken oder Online und des Umgangs mit technischen Geräten und Programmen verfügen. Heutzutage brauchen Lernende ihre bereits soliden Medienkompetenzen zugunsten der Lernprozesse zu investieren. Sie sollen lediglich auf nützliche Portale und Programme hingewiesen werden.

Kompetenzmodelle "setzen methodisch/didaktische Vorgaben, um die gesetzten Erwartungen zu erfüllen" (Beer 2007: 228). Je aktiver Lernende die Bearbeitungsprozesse gestalten, desto beteiligter fühlen sie sich und desto zielbewusster verfahren sie mit der Aufgabenstellung. Das Provozieren ihrer vorhandenen Kompetenzen und die gezielte Bildung neuer Kompetenzen führen automatisch $\mathrm{zu}$ größerer Nachhaltigkeit als der Output jenes Unterrichts, der reines Fachwissen anstrebt. In dieser Studie werden die Schlüsselkompetenzen ausgewählt, die durch das vorgeschlagene Modell zum Einsatz von LK im FSU vernetzt und ausgebildet werden und die die Lernenden auf das Lehramt vorbereiten.

Im Folgenden wird auf die Position der LK im aktuellen, didaktisch-methodischen Diskurs eingegangen, weil die Studie einen kompetenzfördernden Ansatz entwickeln möchte, der im LKU eingeführt wird.

\section{Die LK, ein definitorisches Dilemma}

Die Debatte um den Begriff "LK" erlebt eine lange Tradition der Uneinigkeit. Trotz der prägnanten Bedeutung der LK für den Fremdsprachenerwerb wird sie als "Unfach" (Picht 1980: 270; Schmidt 1980: 289) betrachtet, da der Begriff unter nachhaltigen Schwierigkeiten leidet:

- Schmidt kritisiert die unklare Essenz der LK. Es fehlt ihm die spezifische Wissenschaft, auf die sich die LK bezieht. Hier können Kulturwissenschaft, Geschichte, Geographie, Soziologie, u.a. Wissenschaften in Frage kommen (vgl. Schmidt 1980: 289). Insofern ist die LK "einer der schwierigsten Bereiche des

العدد الرابع والعشرون (الجزء الثانى)

()

مجلة كلية التربية- جامعة عين شمس22 


\section{Dr. Mahmoud Fouad}

Deutschunterrichts," sie umfasst in der Regel "den eigenen Ausgangspunkt und das fremde Ziel, weswegen wir sie interkulturell nennen," und das "unterscheidet sie von anderen Unterrichtsbereichen wie Grammatik oder Phonetik“" (Pauldrach 1992: 15).

- Kritisiert wird die LK, dass sie keinen klaren Ausgangspunkt hat, was Deutschland eigentlich ist: es geht in der LK manchmal von Deutschland als geographische Einheit, aber auch als die deutsche Kultur, die die DACH-Länder umfasst. Subjektive Bezugnahmen bestimmen diesen variablen Deutschlandbegriff (vgl. Pauldrach 1992: 9 ff.).

- Der Einsatz von LK vermisst das didaktisch konturhafte Konzept, so dass jeder das Fach nach individuellem Belieben gestaltet. Daher erscheint die LK als ein "dilettantischer Gemischtwarenladen" (Thoma 1990: 9) zu sein, in dem jede einzelne Lehrperson freie Hand zu haben denkt. "Landeskunde ist so, wie man sie als Lehrperson sieht, versteht bzw. sie verwenden kann: vorwiegend ausschnittweise" (Fleming 2015: 57). Gemäß dieser Definition unterliegt die LK dem persönlichen Verständnis der Lehrkraft, die sich dafür einsetzt, eigene Unterrichtskonzepte zur Themenauswahl und zum didaktischen Einsatz der LK zu entwickeln. Obwohl dieser Ausgangspunkt keine intersubjektive Grundlegung von LK nachweist, versucht er die problematische Position der LK als Fach im FSU zu diagnostizieren, nämlich die fehlenden "Methoden und Strategien, mit denen eigenes Material generiert wird [...] Entscheidend finde ich die Herangehensweise, dass Lernende Produzenten von Unterricht sein müssen, nicht nur Konsumenten" (Fleming 2015: 55), demnach sei generell die methodische Fundierung eines didaktischen Einsatzes von LK, der lernerzentriert und kompetenzfördernd sein soll für das Fach ausschlaggebend.

- Die subjektive und ideologische Betrachtung von LK kann bei der Auswahl landeskundlichen Stoffes nicht ausgeschlossen werden, da die Lehrperson aber auch die Autorenschaft besondere Lernmaterialien auf Kosten anderer auswählt und einführt. Ihre 
Auswahl ist von bestimmten Werten, Neigungen, Interessen und Tendenzen beladen. "Landeskundliches Wissen ist gesellschaftliches Wissen. Es ist darum interpretierbar, abhängig von den Interessen der Informationsquellen" (Pauldrach 1992: 9).

- Die Bezeichnung "LK" setzt den Akzent auf "Land" und steuert damit das Interesse mehr in die Richtung des veränderbaren Geographischen und Politischen, während die permanente, kulturelle Seite des Landes im Begriff nicht hervorgehoben wird, daher wird für den neuen Begriff "Kulturkunde" plädiert (vgl. Grawe 1987: 471). Aber wegen dem Etablieren des Begriffs "LK" hat sich der neue Begriff "Kulturkunde" nicht durchgesetzt.

- Beim didaktischen Einsatz von LK im FSU soll sich weder um "Land" noch um "Kunde" handeln, sondern um zielkulturgerechte und sprachliche Anwendung. „Landeskunde meint alle Bezüge auf die Gesellschaften, deren Sprache im FSU gelernt wird. Solche soziokulturellen Bezüge treten im fremdsprachlichen Curriculum immer [...] auf.“ Dabei ,geht es aber weniger um einen Raum oder eine Region ('Land') als um eine sprachlich artikulierte kulturelle Praxis. Es geht auch weniger um einen abgrenzbaren Wissensbestand ('Kunde'), als vielmehr um eine sprachlich vermittelte interkulturelle Kompetenz" (Buttjes 1989: 112). Gleich diese letzte Definition der LK hebt das Verhältnis der LK mit der Kompetenzbildung im LKU hervor. Im Folgenden werden die bisher herrschenden, didaktischen Ansätze im LKU durchgenommen.

\section{Didaktische Ansätze zum Einsatz der LK im FSU}

Da der Spracherwerb ohne LK kaum vorstellbar ist und die LK dementsprechend ein integraler Teil im FSU bildet, so entwickelt die Sprachlehr- und Sprachlernforschung mehrere Konzepte zum Einsatz von LK im FSU:

- Kramsch setzt sich stark für den kognitiven Ansatz ein, den sie als "Container-Modell" bezeichnet, das die didaktischen Ziele der 
LK als Medium zur Vermittlung von Fakten und Daten, als Medium für "ethnozentrische Verpackung" hervorhebt(Kramsch 1991: 110).

- Im Rahmen des Ansatzes "culture studies" hat Nünning zehn neue Faktoren zur Umgestaltung des LK-Unterrichts von dem kognitiven zum kulturellen Ansatz, aber auch kommunikativen, lernerzentrierten Konzept.

1- von faktenorientierter Wissensvermittlung zum Kultur- unterricht

2- von Lehrer- zur Lernerzentrierung

3- von der Produkt- zur Prozessorientierung

4- von der Lehr- zur Handlungs- und Projektorientierung

5- exemplarisches Lehren und Lernen

6- von `der' Kultur zu den Kulturen

7- vom Wissen über die Zielkultur zum Kulturvergleich

8- Perspektivenvielfalt und Perspektivenwechsel

9- vom Wissenserwerb zur interkulturellen Kommunikationskompetenz

10- vom Sprach- und Literaturunterricht zum historischen Kulturunterricht.

- Raasch entwickelt den Fünf-Kompetenzstufen-Ansatz zum didaktischen Einsatz von LK (Raasch 1997: 71f): Die 1. Stufe weist auf die Vermittlung von Faktenwissen über das Zielland hin. Die 2. ist die kontrastive Stufe, wodurch zwischen der Ziel- und Ausgangskultur verglichen wird. Die 3. Stufe bezweckt die Bildung einer Empathiekompetenz, die man dazu befähigt, Verständnis für die kulturellen Unterschiede zu haben. Dann folgt die 4. Stufe der interkulturellen Kompetenz, die die kulturellen Unterschiede überwindet und Kooperation zwischen den Kulturen bestrebt. Die 5 . Stufe sei die Interkulturalität selbst, die nicht mehr von Kulturen ausgeht, sondern von einer gemeinsamen, übergreifenden, menschlichen Kultur.

- In seiner Erwägung rekapituliert Neuner die bisherige Position der LK im FSU, die zwei Faktoren unterliegt: den 
Kompetenzförderung im Landeskundeunterricht

Rahmenbedingungen und den einzusetzenden, didaktischen Konzepten (vgl. Neuner 1994: 17f):

Die Rahmenbedingungen der LK im FSU umfassen übergreifende gesellschaftlich-politische Faktoren wie das Verhältnis im eignen Land zum Zielsprachenland, übergreifende intentionelle Faktoren wie die herrschenden, pädagogischen Konzepte, die fachdidaktischen Zielsetzung im FSU und letztlich die Konzepte der Unterrichtsgestaltung.

Die didaktischen Konzepte der LK im FSU sind nach Neuner die kognitiven, kommunikativen und interkulturellen Ansätze, welche sich prinzipiell im LKU vermischen können, doch ihre Ziele und Inhalte variieren sich.

\begin{tabular}{|l|l|l|l|}
\hline $\begin{array}{l}\text { didakt. } \\
\text { Konzept }\end{array}$ & $\begin{array}{l}\text { Kognitiver } \\
\text { Ansatz }\end{array}$ & $\begin{array}{l}\text { Kommunikativer } \\
\text { Ansatz }\end{array}$ & $\begin{array}{l}\text { Interkult. } \\
\text { Ansatz }\end{array}$ \\
\hline $\begin{array}{l}\text { didakt. } \\
\text { Ort }\end{array}$ & $\begin{array}{l}\text { eigenes Fach/ } \\
\text { selbständige } \\
\text { Lerneinheit }\end{array}$ & im FSU & im FSU \\
\hline $\begin{array}{l}\text { Über- } \\
\text { geordnete } \\
\text { S } \\
\text { Ziel }\end{array}$ & $\begin{array}{l}\text { Wissen: } \\
\text { Systema- } \\
\text { tische } \\
\text { Kenntnisse } \\
\text { über } \\
\text { Kultur und } \\
\text { Gesellschaft }\end{array}$ & $\begin{array}{l}\text { Kommunikative } \\
\text { Kompetenz: } \\
\text { Verständigung } \\
\text { ohne } \\
\text { Missverständ- } \\
\text { nisse }\end{array}$ & $\begin{array}{l}\text { Kommuni- } \\
\text { kative } \\
\text { und kulturelle } \\
\text { Kompetenz: } \\
\text { sich und andere } \\
\text { besser verstehen }\end{array}$ \\
\hline Inhalte & Soziologie & $\begin{array}{l}\text { Wie Leute } \\
\text { wohnen, sich } \\
\text { erholen, } \\
\text { miteinander } \\
\text { kommunizieren, } \\
\text { am Gemeinwesen } \\
\text { teilnehmen? } \\
\text { (kulturelle } \\
\text { Tradierung) ... }\end{array}$ & $\begin{array}{l}\text { Alle Repräsen- } \\
\text { tationen } \\
\text { der Zielkultur im } \\
\text { Unterricht und } \\
\text { ihre Bedeutung } \\
\text { innerhalb der } \\
\text { Zielkultur und für } \\
\text { den Lernenden }\end{array}$ \\
& Politik & Wirtschaft \\
& Kultur & Geschichte &
\end{tabular}

العدد الرابع والعشرون (الجزء الثانى)

()

مجلة كلية التربية- جامعة عين شمس 
Dr. Mahmoud Fouad

\begin{tabular}{|l|l|l|l|}
\hline $\begin{array}{l}\text { didakt. } \\
\text { Konzept }\end{array}$ & $\begin{array}{l}\text { Kognitiver } \\
\text { Ansatz }\end{array}$ & $\begin{array}{l}\text { Kommunikativer } \\
\text { Ansatz }\end{array}$ & $\begin{array}{l}\text { Interkult. } \\
\text { Ansatz }\end{array}$ \\
& & ALLTAGS- & FREMD- \\
& $\begin{array}{l}\text { LANDES- } \\
\text { KILD }\end{array}$ & $\begin{array}{l}\text { GESUR/ } \\
\text { GESPÄCHS- } \\
\text { THEMEN }\end{array}$ & STEHEN \\
& & THEMER- & \\
\hline
\end{tabular}

(vgl. Neuner 1994: 17f):

Nach diesem Überblick über die definitorische und didaktischmethodische Problematik der LK möchte die Studie auf die Stellung der LK im Lehrplan der Deutschabteilung der Pädagogischen Fakultät der Universität Ain Shams analytisch eingehen.

\section{LK im Lehrplan des untergradualen Studiums an der Deutschabteilung der Pädagogischen Fakultät}

Die Deutschabteilung der pädagogischen Fakultät bildet Deutschlehrerstudierende aus, für die Deutschlehrerausbildung wird die LK im Lehrplan als eigenständiges Fach eingesetzt.

\begin{tabular}{|l|l|l|l|}
\hline Stj. & Sem. & Code & Bezeichnung \\
\hline 1. & 1 & G115 & $\begin{array}{l}\text { Übungen zur interkulturellen Kommunikation }{ }^{1} \text { oder } \\
\text { Einführung in die deutsche Kultur I } \\
\text { (Wahlfächer, nur eins wird ausgewählt und eingesetzt) }\end{array}$ \\
\cline { 2 - 5 } & 2 & $\begin{array}{l}\text { G125 } \\
\text { G126 }\end{array}$ & $\begin{array}{l}\text { Übungen zur interkulturellen Kommunikation oder } \\
\text { Einführung in die Kultur II } \\
\text { (Wahlfächer, nur eins wird ausgewählt und eingesetzt) }\end{array}$ \\
\hline 2. & 1 & G212 & Kultur- und LK: Alltagskultur \\
\cline { 2 - 5 } & 2 & G222 & Kultur- und LK: Geschichte der DACHL \\
\hline 3. & 1 & G312 & Deutsche Philosophie- und Kulturgeschichte \\
\cline { 2 - 5 } & 2 & G325 & Kultur- und LK: Gesellschaftliche Institutionen oder \\
\hline
\end{tabular}

1 G115 und G125 haben dieselbe Bezeichnung und werden jedoch in zwei unterschiedlichen Semestern eingesetzt.
العدد الرابع والعشرون (الجزء الثان) 2018
()
مجلة كلية التربية- جامعة عين شمس27 
Kompetenzförderung im Landeskundeunterricht

\begin{tabular}{|l|l|l|l|}
\hline \hline Stj. & Sem. & Code & Bezeichnung \\
\hline & & G326 & $\begin{array}{l}\text { Das deutsche Bildungssystem - vergleichende Studie } \\
\text { (Wahlfächer, nur eins wird ausgewählt und eingesetzt) }\end{array}$ \\
\hline 4. & 1 & G412 & Kultur- und LK: Kulturkontakt \\
\cline { 2 - 4 } & 2 & G422 & Problemfelder deutscher Kultur \\
\hline
\end{tabular}

Der Ausgangspunkt im Lehrplan, dass die LK als eigenes Fach eingesetzt und nicht in den FSU oder für dessen beabsichtigten Fertigkeiten integriert wird, betont die prinzipielle, kognitive Zielsetzung der LK im Lehrplan (s. o. Neuners didaktische Konzepte). Dabei repräsentieren die Fächer die drei von Neuner erwähnten, didaktischen Ansätze. Im Folgenden werden die Fächer je nach dem didaktischen Ansatz zugeordnet. Hierbei wird die Kursbeschreibung der Fächer näher betrachtet, um zu überprüfen, ob die Bezeichnung des Faches dessen Inhalte widerspiegelt.

Folgende Fächer der LK im Lehrplan werden Neuners didaktischen Ansätzen zugeordnet.

\begin{tabular}{|l|l|l|}
\hline \multicolumn{3}{|c|}{ Didaktischer Ansatz } \\
\hline Kognitiv & kulturell, kommunikativ & interkulturell \\
\hline & G116 \\
& G126 & G115 \\
\hline G222 & G212 & G125 \\
\hline G312 & G325 & \\
\hline & G412 & G326 \\
\hline & G422 & \\
\hline
\end{tabular}

kognitiv: 2 Pflichtfächer

kulturell, kommunikativ: 3 Pflichtfächer und 3 Wahlfächer, Die Wahlfächer, die kommunikativ konzipiert sind, können von der Lehrperson ausgewählt werden, sie kann aber auch diese Fächer nicht auswählen und anstatt deren die anderen, verfügbaren Fächer, die interkulturell konzipiert sind, denn in einem Semester wird nur eines der beiden Wahlfächer ausgewählt und eingeführt. Mit anderen Worten sind es Wahlfächer für die Lehrperson und nicht für die Lernenden.

$$
\begin{aligned}
& \text { العدد الرابع والعشرون (الجزء الثانى) } 2018 \\
& \text { مجلة كلية التربية- جامعة عين شمس28 }
\end{aligned}
$$


interkulturell: wieder 3 Wahlfächer, die eingeführt werden können aber nicht müssen. Somit kann dieser Ansatz überhaupt ausgeschlossen werden, wenn die drei dafür geeigneten Fächer von den Lehrpersonen zum Einsatz nicht ausgewählt werden.

Obwohl man zwischen den Fächern 115-116, 125-126 und 325326 auswählen kann, unterliegt jeder Teil der drei Fächerpaare einem unterschiedlichen Ansatz. Beispielsweise kann sich eine Lehrperson in einem Studienjahr für 115 und sich eine andere im darauffolgenden Jahr für 116 entscheiden. Demgemäß beruht der Lehrplan auf keinem klaren, didaktischen Konzept zum Einsatz der LK. Auch ein Ansatzpluralismus ist kein Ziel des Lehrplans, da der interkulturelle Ansatz, wie eben erklärt, nur in einem Wahlfach einsetzbar ist.

Die didaktisch-methodische Unübersichtlichkeit im Lehrplan bezüglich der LK bewegt die vorliegende Studie, ein didaktisches Modell zum Einsatz der LK im FSU in Ägypten vorzuschlagen, das abgesehen von Bezeichnung und Inhalt des Faches ein allgemeingültiges Konzept zum LK-Einsatz entwirft und sämtliche kognitiven, kulturell-kommunikativen und interkulturellen Zielsetzungen der LK berücksichtigt und sie im FSU integriert. Ein Modell, das die Kompetenzen der Lernenden anspricht, provoziert und weiterentwickelt. Damit ist das Modell fächerübergreifend, mit anderen Worten kann es in anderen Fächern eingesetzt werden. Aber unter den DaF-Fächern erlaubt besonders die LK die Bildung der meisten Kompetenzen, die im und durch den FSU entfaltet und fortgebildet werden können. Dazu erfordert das definitorisch und didaktisch-methodisch heikle Wesen der LK eine neue Konzeption des Faches im FSU, die im Folgenden dargestellt wird.

\section{Die LK im FSU}

Die bisherige Position der LK im FSU bewegt 1990 eine vom Fachverband "Moderne Fremdsprachen" und Goethe-Institut beauftragte Kommission aus den DACH-Ländern dazu, die Grundsätze für die Rolle der LK sowie für ihre methodischen

$$
\begin{aligned}
& \text { العدد الرابع والعشرون (الجزء الثانى) } 2018 \\
& \text { مجلة كلية التربية- جامعة عين شمس29 }
\end{aligned}
$$


Kompetenzförderung im Landeskundeunterricht

Realisierungen im Deutschunterricht abhandeln sollte. Der Ausschuss kommt $\mathrm{zu}$ der folgenden Auffassung von LK: "Landeskunde im Fremdsprachen ist ein Prinzip, das sich durch Kombination von Sprachvermittlung und kultureller Information konkretisiert und durch besondere Aktivität über den DU hinaus wirken soll, z. B. durch Austausch und Begegnung. Insofern ist LK kein eigenes Fach" (ABCD-Thesen: 306). Diese synthetische Vorstellung greift auf die kulturelle und informative Dimension der LK aber auch auf die fächerüberschreitende Natur der LK über. Der Aspekt der erlebten LK wird außerdem hervorgehoben. Aus den Kommissionsbesprechungen resultieren 22 Grundsätze zur didaktischen Konzeption der LK für den FSU.

\subsection{Die Kompetenzbildung gegen den kognitiven Ansatz im LKU}

An der Lehrerausbildung stellen die ABCD-Thesen folgende Aufgaben: "eine entsprechende Didaktik und Methodik landeskundlichen Unterrichts" (ebd.: 307). Ein vollständiges Landesbild kann der LKU nicht vermitteln. Das bedeutet, dass der kognitive Ansatz, der hauptsächlich Faktenwissen bezweckt, im LKU seine Lernziele nicht völlig erfüllen kann. In diesem Bezug betont der 2. Grundsatz der ABCD-Thesen diesen Aspekt der exemplarischen Darstellung von landeskundlichem Faktenwissen. "Landeskunde wird dynamisch und prozesshaft gesehen. Auf die Vollständigkeit der Informationen im Hinblick auf hypothetisches Landesbild wird bewusst verzichtet" (ebd.: 306). Der Sinn des kognitiven Ansatzes und seine Ziele werden zurecht in Frage gestellt.

Daher sollte sich der LKU andere Ziele setzen als die Vermittlung vom Faktenwissen. Die übrigen, kommunikativen und interkulturellen Ansätze des LKUs setzen die Verfügbarkeit bestimmter Kompetenzen bei den Lernenden voraus, um ihre Effizienz erst $\mathrm{zu}$ erreichen, wie die Kompetenz zur sprachlich adäquaten Kommunikation, und die zur Analyse der zielkulturellen

العدد الرابع والعشرون (الجزء الثانى)

()

مجلة كلية التربية- جامعة عين شمس30 
Wirklichkeit und zum Perspektivenwechsel. Die Kompetenzbildung als Ziel des LKUs hebt die 4. These der ABCD-Thesen ganz evident hervor. "Primäre Aufgabe der LK ist nicht die Information, sondern die Entwicklung von Fähigkeiten, Strategien und Fertigkeiten im Umgang mit fremden Kulturen" (ebd.: 306).

Eine weitere Betonung des kompetenzbildenden Ansatzes lässt sich von der 6. These erschließen: "Landeskunde steht in engem Zusammenhang mit dem Spracherwerb. Rücksichtnahme auf die eingeschränkte Sprachfähigkeit der Lernenden und die sprachliche Progression fordern didaktische Vereinfachung, dürfen aber nicht zu Simplifizierung, Verniedlichung, Vergröberung und Verzerrung führen"(ebd.: 307). Obwohl die LK ein Bestandteil des Spracherwerbs ist, kann das Problem des sprachlich nichtangemessenen Niveaus der Lernenden durch didaktische Manöver bewältigt werden. Daraus ist zu erschließen, dass der LKU im Wesentlichen nicht auf Sprachförderung zielt, sondern er ist ein "Prozess er aktiven Auseinandersetzung mit der fremden Kulturen", dabei ist es wichtig, die Lernenden "zu schöpferischer Arbeit anzuregen und zu ermutigen" (ebd.: 307).

Resümierend ist es einleuchtend, dass der LKU "Entwicklung von Fähigkeiten", "aktive Auseinandersetzung" und "schöpferische Arbeit" (ebd.: 306f) bezweckt, nicht aber die informative Vermittlung, was ausgesprochen den Einsatz des kompetenzförderenden Ansatzes auf Kosten des kognitiven Ansatzes im LKU empfiehlt. Auch die kommunikativen und interkulturellen Ansätze streben apriorisch die Bildung der beiden Kompetenzen an.

Als erster Schritt zur Erstellung dieses kompetenz- fördernden Ansatzes wäre die angemessene Auswahl von Materialien, die einerseits für das jeweils Fachwissen andererseits für die Kompetenzbildung geeignet sein sollen.

\subsection{Kriterien der Auswahl landeskundlicher Materialien}

Inhalt des landeskundlichen Stoffes kategorisiert sich in drei Hauptgruppierungen (vgl. Bischof 1987):
العدد الرابع والعشرون (الجزء الثانى) 2018
()
مجلة كلية التربية- جامعة عين شمس31 
a. Faktenwissen und Daten (was, wie gesagt, überholt wird.)

b. Authentische Situationen und kommunikative Alltagsthemen der zielkulturellen Angehörigen

c. Institutionelle Apparate und gesellschaftliche Ordnung

Die selektierten, landeskundlichen Materialien sollen klaren und durchdachten Kriterien unterliegen. "Gerade exemplarisches Lernen, das nicht auf die Totalität seines Gegenstandes abhebt, ist auf begründete und zuverlässige Auswahl angewiesen" (Buttjes 1989: 116). Dies betonen auch die ABCD-Thesen der LK: Die Materialien sollen möglichst so authentisch sein, dass die Neugier und Lust der Lernenden auf Entdeckung geweckt werden sollen. (These 10, ABCD-Thesen: 307).

Buttjes geht davon aus, dass Inhalte der LK an und für sich ausgewählt werden sollen, darauf folgend sollen sie didaktischmethodisch bearbeitet und eingeführt werden."Eine einfache Ableitung landeskundlicher Lerninhalte ist weder aus den Interessen oder der Kommunikationsfähigkeit der Lernenden noch aus der Beschreibung der Zielgesellschaft oder der Bestimmung kultureller Interferenzen möglich" (Buttjes 1989: 116). Zur gerechten Auswahl landeskundlicher Inhalte bestimmt er folgende Ebenen:

a. Realitätsebene: Der Stoff enthält quellensichere Realien.

b. Konkretionsebene: Der Stoff lässt sich in sprachliche, konkrete Situationen verwandeln. Der LKU soll zulassen, dass subjektive und emotionale Einstellungen der Lernenden zur Zielkultur frei ausgedrückt werden (These 17: 307).

c. Transferebene: Das landeskundlich behandelte Thema lässt sich in die eigene Gesellschaft übertragen.

d. Identifikationsebene: Der Stoff hat einen interkulturellen Bezug. (Buttjes 1989: 116)

Andere Studien haben weitere Kriterien bei der Stoffauswahl berïcksichtigt:

1. Institutionelle Rahmenbedingungen: Kursbeschreibung, Curricula, Evaluationsordnung, Veranstaltungszahl und -dauer im Semester. 


\section{Dr. Mahmoud Fouad}

2. Thematik: Auswahl der Texte soll auch das intendierte, didaktische Modell im Unterricht berücksichtigen. Die landeskundlichen Texte sind "immer Bestandteile komplexer kommunikativer Handlungsspiele" (Schmidt 1980: 293). Aufgrund ihrer gesellschaftlichen und thematischen Implikate, ihres sprachlichen Niveaus und ihrer Aktualität im zielkulturellen Kontext soll diese kommunikativen Handlungen für die Lernenden geeignet sein.

3. Zielgruppenkompetenzen: Eine wichtige Frage bei der lernerorientierten Textauswahl ist: Was die Zielgruppe weiß und kann bzw. wissen oder können soll. "Bei dem Können und Wissen werden lexikalische, grammatische, stilistische, sowie produktive bzw. rezeptive Kompetenzen der Lernenden in der Zielsprache berücksichtigt. [...] Beim Sollen handelt es sich um zu erreichenden Fähigkeiten und Qualifikationen, die durch den institutionellen Rahmen vorgegeben sind" (Dzykovych 2016: 42).

Eine weitere Frage, die für die Zielgruppe relevant, ob für sie bestimmte lernpsychologische, soziale, religiöse, moralische Lernhindernisse bzw. Tabus haben.

4. Didaktik, Methodik, Lehrperson: Didaktisches und methodisches Konzept, lernerorientierte Sozialformen, der nötige Sachverstand, plausible Lehr- und Lernziele, vorhandene Hilfsmittel und verfügbare Freiräume gehören zu den Grundvoraussetzungen der Lehrperson, die den landeskundlichen Stoff auswählt.

Diese Faktoren schließen andere nicht aus, da es sich bei der LK um einen variablen Bereich handelt, daher "scheint es nicht möglich zu sein, einen allgemein verbindlichen Stoffkanon aufzustellen" (Dzykovych 2016:40).

Hauptsächlich bei der Stoffwahl ist die Berücksichtigung, dass der ausgewählte Stoff erstens dem didaktischen Ansatz zum LKEinsatz entspricht, zweitens über das Informative hinausgeht und drittens die Kompetenzbildung bei den Lernenden bezweckt. Auch die intendierten Kompetenzen sollen die kognitive, kommunikative und interkulturelle Kompetenz einbeziehen. Aus diesem letzten Punkt ist zu erschließen, dass das in dieser Studie entworfene

العدد الرابع والعشرون (الجزء الثان) 2018

()

مجلة كلية التربية- جامعة عين شمس33 
Modell zum Einsatz von LK im FSU lernerzentriert, handlungs- und produktionsorientiert zu bezeichnen ist und dass dieses Modell alle didaktischen Konzepte des LKUs berücksichtigt und mit einbezieht. Eine gewisse Verarmung für den LKU wäre die Anwendung eines einzigen Konzepts. LK ist ein interdisziplinäres Fach, das Methodenvielfalt beansprucht, die u.a. selbständiges und autonomes Lernen bezweckt (vgl. These 18, ABCD-Thesen: 307). Abgesehen von dem landeskundlichen Stoff erlaubt der LKU immens mannigfache Möglichkeiten zur Entfaltung der Kompetenzen der Lernenden. $\mathrm{Ob}$ es im LKU um Faktenwissen oder prozedurales Wissen geht, eignet sich das im Folgenden vorgeschlagene Modell zum didaktischen Einsatz im LKU mit dem Ziel auf Kompetenzbildung bzw.-fortbildung.

\section{Didaktischer Einsatz der LK im FSU zur Kompetenzförderung}

Rahmenbedingungen: Im WS 2017/18 wird im Fach LK für das 2. Studienjahr "Kultur- und Landeskunde: Alltagskultur. G212" das durch diese Studie vorgeschlagene Modell an der Deutschabteilung der Pädagogischen Fakultät der Universität Ain Shams in Kairo/Ägypten eingebracht. Die Studierenden erfahren die LK in diesem Semester erstmal als selbständiges Fach. LK ist zwar im 1. Studienjahr in den Sprachkurs integriert, bleibt lediglich als offizielle Bezeichnung in der Satzung zugunsten der Förderung der sprachlichen Fertigkeiten, die durch das ganze Studienjahr im absoluten Mittelpunkt des Interesses stehen. Die runden Tische gewährleisten eine interaktionsfreundliche, nichtlinienförmige Gestaltung des Seminarraums.

Die Gruppe: Die Studierenden besuchen die Universität mit geringer Deutschkenntnis (Niveau A1/1), die sie sich in den staatlichen Oberschulen angeeignet haben, zu diesem Zeitpunkt haben sie das Niveau A2/B1. Aus abteilungsinternen und räumlichen Gründen wird die Zielgruppe in zwei große Gruppen eingeteilt, je aus 35

العدد الرابع والعشرون (الجزء الثانى) 2018

مجلة كلية التربية- جامعة عين شمس34 
Studierenden bestehend. Jede Gruppe bekommt von der gleichen Lehrperson den gleichen inhaltlichen Input.

Materialien: Gemäß dem Curriculum für "Alltagskultur" wird eine Liste mit aktuellen Themen erstellt, über sieben davon sollten die Lernenden gleich in der ersten Sitzung einen Konsens haben. Das Semester dauert vierzehn Wochen, so sind zwei Sitzungen für jedes Thema vorgesehen. Für jedes Thema sind so viele Materialien wie die Anzahl der Arbeitsgruppen ausgewählt, so dass sich jede Gruppe mit einem eigenen, landeskundlichen Material beschäftigt. Die Kriterien zur Auswahl landeskundlicher Materialien, die in dieser Studie ausgeführt wurden wie Authentizität, Konkretion, Tranferierbarkeit etc., habe ich hier voll und ganz berücksichtigt.

Die Probandengruppe: Jede Teilgruppe teilt sich in sechs Arbeitsgruppen auf. Die Lehrperson führt das Modell an das ganze 2. Studienjahr durch, analysiert sämtliche Aufgaben aller Arbeitsgruppen und misst die Bildung und Entwicklung der Kompetenzen bei allen Lernenden anhand ihrer Aufgaben. Aus ökonomischen Gründen sollte die Studie eine bestimmte Arbeitsgruppe auswählen, die als Versuchsgruppe dient und anhand deren Aufgabe der Prozess der Kompetenzbildung hiermit dargestellt und analytisch, repräsentativ ermittelt wird. Die Auswahl trifft besonders die Arbeit dieser Versuchsgruppe aus den folgenden Gründen:

Die Studierenden hatten die Freiheit, sich kleineren, aus fünf bis sechs Mitgliedern bestehenden Arbeitsgruppen anzuschließen. Die letzten fünf Studierenden haben keiner Gruppe zugesellt. Sie mussten dann eine Zwangsgruppe bilden. sich aus den fünf übrigen Studierenden, die keiner Gruppe zugesellt haben. Diese Rahmenbedingung, dass man seine Kollegen nicht selbst auswählt, kann man eventuell im beruflichen Leben begegnen.

Sie hatte dazu große Probleme beim Zeitmanagement. Erst nach fünf Wochen hat diese Gruppe gelernt, die Zeit vernünftig zu verwalten und die Aufgaben zeitmäßig zu erledigen.

Alle Mitglieder dieser Gruppe haben gemäß den Prädikaten im ersten Studienjahr bescheidene Noten erhalten, so dass eine
العدد الرابع والعشرون (الجزء الثاني) 2018
()
مجلة كلية التربية- جامعة عين شمس 
Kompetenzförderung im Landeskundeunterricht

Entwicklung im Niveau der Gruppenmitglieder leicht zu bemessen ist.

\subsection{Beschreibung des didaktischen Modells zum Einsatz der LK zur Kompetenzbildung}

In jeder Sitzung wird eine Kette von Aufgaben an den einzelnen Gruppen gestellt. Diese Aufgaben sollen die Lernenden unter Zeitdruck setzen, die Kompetenzen der einzelnen Mitglieder provozieren und neue Kompetenzen bei ihnen didaktisch gezielt bilden.

\subsubsection{Aufgabe zur Bildung der Lese-, Textbearbeitungs-, Teamwork-, Zeitmanagements-, $\quad$ Problemlösungs-, und Moderationskompetenz:}

Jede Gruppe soll den landeskundlichen Text innerhalb eines bestimmten Zeitraums, der der Länge und dem Schwierigkeitsgrad der Texte kaum passt, lesen und ihn auf seine inhaltlichen bzw. informativen Stichpunkte rekapitulieren. Jede Gruppe bekommt sämtliche Texte, die in den LKU eingeführt werden, behandelt aber nur einen Text davon, wobei der eine Text nur einmal ausgewählt werden darf. Die landeskundlichen Inhalte werden dann im Plenum von den anderen Gruppen gehalten, so wird das landeskundliche Thema durch die Zusammenfassungen aller Texte fachlich erschöpfend gedeckt. Dann werden diese Schlussfolgerungen im Plenum vorgehalten, indem jedes Mitglied in der Gruppe zu Wort kommen soll.

Die Lehrperson soll hierbei überprüfen, ob die Lernenden die im Text beinhaltenen, landeskundlichen Informationen vollständig herausgearbeitet haben, um die Lese- und die Textbearbeitungskompetenz auf Probe zu stellen.

Die Lernenden sollen dazu ihre Kollegen im Plenum moderierend konfrontieren, um die Ergebnisse der Text- erschließung zu halten. Die Lehrperson soll nicht nur die Richtig- und Vollständigkeit der

العدد الرابع والعشرون (الجزء الثانى) 2018

()

مجلة كلية التربية- جامعة عين شمس 
Gruppenberichte überprüfen, sondern auch die Aussprache und die Vortragstechnik der Lernenden in Betracht ziehen.

\subsubsection{Aufgabe zur Bildung der Textsorten-, Kreativitäts-,} Schreib-, Ausdrucks-, Präsentations-, kommunikative und interkulturelle Kompetenz

Gleich im Anschluss der Gruppenberichte im Plenum wird eine neue Aufgabe gestellt: Das Konzept des "prozessorientierten Schreibens" (Reder 2013: 181), das sich in sechs Phasen vollzieht (1) Impulse geben, (2) Ideen sammeln, (3) Planen, (4) einen Ersttext erstellen, (5) den Text überarbeiten und (6) den Text präsentieren, wird in dieses Stadium des Modells übertragen.

Zur Bildung der Textsorten-, Kreativitäts-, Schreib-, kommunikativen und interkulturellen Kompetenz sollen die Gruppen den behandelten Text in szenische Gespräche transformieren, woran alle Mitglieder der jeweiligen Gruppen Rolle haben sollen. Die Transformation der Texte in andere Textsorte nämlich in Szenen und die Bewusstmachung, dass die Lernenden selbst einen neuen Text erstellen, hebt die Transferierbarkeit und die Mobilität auf Textebene hervor (vgl. Die Schlüsselkompetenzen unter 2.2. und zum Begriff "Mobilität", vgl. Beck 1993: 17f). Die Mobilität gilt eigentlich als das Hauptziel der Kompetenzbildung. Das Modell bezweckt die Entfaltung der Kreativitäts- wie kommunikativen Kompetenz bei den Lernenden, indem sie die aus dem Text entnommenen Informationen über das landeskundliche Thema ins Gespräch integrieren, das sich zwischen Partnern aus Deutschland und Ägypten abspielt. Dadurch soll ihre interkulturelle Kompetenz geschult werden, wobei beide Kulturen im Gespräch in Berührung kommen. Die kulturellen Unterschiede bzw. Gemeinsamkeiten werden durch das Nebeneinander im Gespräch begreifbar gemacht. Die verfassten Szenen der Arbeitsgruppen werden im Plenum vorgespielt. Hier wird auch die Präsentationskompetenz angesprochen. 


\section{Kompetenzförderung im Landeskundeunterricht}

\subsubsection{Auswertung des Experiments}

Erste Aufgabe: In der elften Woche behandelte der LKU das Thema "Essen und Trinken". Die Versuchsgruppe wählte einen Text, mit dem Titel "Essen" aus, der aus der Webseite "slowgerman" heruntergeladen wird. Die Lektüre und die stichwortartige Zusammenfassung des Textes sollten in 20 Minuten erfolgen.

Es hat sich ergeben, dass die einzelnen Gruppen in den ersten Sitzungen aus zeitlichen Gründen die Aufgabe nicht völlig erledigen konnten, bei der Versuchsgruppe hat es die ersten fünf Wochen gedauert, bis sie sich eine Strategie der Arbeitsteilung entwickelt hat: Jedes Mitglied übernimmt einen Textausschnitt und so verschafft sich die Gruppe mehr Zeit zur Textbearbeitung, dann werden die einzelnen Teile nebeneinander zu einem ganzen Output der Gruppe avanciert. Demnach zeigt die Teamworkkompetenz eine gewisse Entfaltung. In derselben Woche hat die Versuchsgruppe alle Inhalte des Textes mit einer einzigen Ausnahme herausgearbeitet.

Jedes Mitglied in der Gruppe sollte beim Referieren der zusammengefassten Textinhalte zu Wort kommen, so sollten alle Mitglieder an der Textbearbeitung teilhaben, dazu haben sie ihre Moderationskompetenz am Ende des Versuchs immens entwickelt und ihre Gehemmtheit, vor Publikum zu sprechen, umsichtig überwunden. Die Gruppenmitglieder sprechen ja nicht fließend, aber das kompetenzförderende Modell setzt sich andere Prioritäten ein, nämlich die Förderung von DaF-übergreifenden Kompetenzen wie die Problemlösungs-, Teamwork- und Zeitmanagementkompetenz, die bei der Versuchsgruppe ganz evident gefördert und entfaltet werden.

Zweite Aufgabe: Bis zur 8. Woche hatte die Versuchsgruppe den Transformationsprozess des eingeführten Textes in ein Gespräch als schwierig betrachtet. Sie hat dann die Szene als eigenständige Form mit erfundener, situativer Handlung zu verstehen begonnen, in der

العدد الرابع والعشرون (الجزء الثانى) 2018

مجلة كلية التربية- جامعة عين شمس38 
die landeskundlichen Informationen paraphrasiert werden. So hat die Gruppe sich von der Struktur des eingeführten Textes befreit.

Die Versuchsgruppe erfindet folgende Situation. Teile der Aufgabe wie im Original werden im Folgenden dargestellt:

Zwei ägyptische Jugendliche (̈̈1, Ä2), die Deutsch studieren, treffen sich mit einer deutschen Familie - Vater (V), Mutter (M) und einem jungen Sohn (S) in Al-Hussein, alle gehen nach einer kurzen Bekanntmachung zusammen essen.

Ä2: Wollen Sie gutbürgerlich, ägyptisch essen oder was anderes?

$V: \quad$ Wir, Deutsche, essen ganz gern Multikulti.

Ä1: $\quad$ Was bedeutet das? Ist Multikuli eine deutsche Spezialität?

S: $\quad$ Das heißt. Wir probieren gern alles Neue, wir essen gern italientisch, griechisch, chinesisch, türkisch usw.

Ä2: $\quad$ Wir essen heute Kebab.

M: $\quad$ Das haben wir in Deutschland. Dönerkebab, es ist türkisch und nicht ägyptisch.

Ä2: $\quad$ Kebab ist bei uns etwas anderes. Es ist gegrilltes Fleisch.

Ä1: $\quad$ Aber auch gegrillte Leber und Frikadellen.

S: $\quad$ Oh. Interessant, ich möchte mal Koushari essen.

$\ddot{A}: \quad$ und was essen und trinken Deutsche gern?

M: Im Frühstück trinken wir Kaffe und essen Käse, Eier, Marmelade, Butter und manchmal Müsli und Jogurt.

$V: \quad$ Beim Mittagessen sind wir meistens unterwegs, in der Arbeit oder in der Schule. So essen wir am Imbiss Wurst oder Salat mit Ei.

M: $\quad$ Am Wochenende gehen wir meistens ins Restaurant, essen Suppe, Steak, Schnitzel, Eintopf, Klöße usw.

S: Hi, Mutti. Vorspeise, Hauptgericht und Nachtisch, das ist alles traditionell. Ich mag Fast-Food, Hamburger, Pommes, Cola und so.

Die ausgewählte, szenische Situation, in der die Kulturen zusammenkommen, übersetzt die Kreativität der Lernenden auf
2018
العدد الرابع والعشرون (الجزء الثانن)
()
مجلة كلية التربية- جامعة عين شمس39 
inhaltlicher Ebene. Anfangs hat die Gruppe uneingeschränkt ihre Gespräche in Frage-Antwort-Form ausgedrückt, es hat etwa 8 Sitzungen gebraucht, bis sie innovative Gesprächshandlungen verfasst hat, z.B. lustige Schwänke und humorvolle, kulturelle Missverständnisse, wie die Verwechselung von Kebab und Dönnerkebab und von Multikulti als deutscher Spezialität.

Die Kreativitäts-, Ausdruck- und Schreibkompetenzen werden in diesem Schritt des Verfahrens geschult, und je mehr sich die Lernenden mit dem Transformationsprozess des Faktenwissens in nachgestellte, kommunikative Lernsituationen auseinandersetzen, desto innovativer und authentischer werden die Gespräche.

Die Aufgabe hebt die interkulturelle Kompetenz hervor, indem sie das Zusammenkommen von Ägyptern und Deutschen voraussetzt. Essgewohnheiten der beiden Kulturangehörigen stehen in der Aufgabe nebeneinander, und man beginnt Verständnis für fremdkulturelle Unterschiede $\mathrm{zu}$ haben, wie die Deutschen im Gespräch offen für alle fremden Spezialitäten sind (der Sohn möchte noch ägyptisches Koushari essen.)

Ein besonders zu beachtendes Ergebnis hat mit der Entwicklung der Kreativitäts-, Problemlösungs- und kommunikativen Kompetenz zu tun. Das ist aus der nächsten Gesprächssequenz zu entnehmen. Die Versuchsgruppe sollte im Plenum ihre Szene vorspielen, aus Zeitknappheit hat sie aber die Szene nicht zu Ende verfasst, so sollten ihre Mitglieder in dieser ungeplanten Situation spontan handeln und frei die Kommunikationssituation erfolgreich zu Ende improvisieren, so entsteht die folgende Sequenz.

Ä1: $\quad$ eh ... Jugendliche auch in Ägypten lieben Fastfood.

Ä2: $\quad$ Wir essen so bei Macdonalds, wenn wir ausgehen.

M: $\quad$ Jugendliche sind so überall. Hm.

V: $\quad$ Gut. Jetzt bestellen wir.

(Die Gruppenmitglieder sitzen am Tisch.)

S: $\quad$ Oh, lecker. Ägyptisches Essen.

العدد الرابع والعشرون (الجزء الثانى)

()

مجلة كلية التربية- جامعة عين شمس40 
Dieser Fortschritt und die Kompetenz, in unerwarteten Situationen angemessen $\mathrm{zu}$ agieren, zeigen die Effizienz des Modells zur Kompetenzbildung. Nicht nur die Entwicklung der kommunikativen Kompetenz zeigt sich präsent, sondern auch die interkulturelle, indem die Mitglieder meinten, dass Jugendliche überall gleich sind. Hier wird von einer menschlichen Kultur bewusst gemacht und nicht von Eigenem und Fremdem. Auch die Entfaltung der Problemlösungs- und Teamworkkompetenz kann man gut erkennen, indem jedes Mitglied das andere nicht im Stich lässt und am roten Faden im Gespräch teilhat. Hochzuschätzen ist, dass die Gruppe beim Weiterführen der Szene nicht stockt, wenn das vorbereitete Gespräch aus zeitlichen Gründen keine komplette Handlung wiedergab.

\section{Ergebnisse der Studie}

Die Ergebnisse der Studie rekapitulieren sich in den folgenden Aspekten.

Hochschulausbildung: Die Bologna-Prozesse setzen den didaktisch-methodischen Akzent im universitären Kontext auf die Kompetenzförderung, die die Flexibilität in der Praxis anstrebt. Nicht mehr die Vermittlung von Fachwissen ist das einzige Ziel der neuen Hochschulausbildungsvision, sondern die Transferierbarkeit und Mobilität, zu der die kognitiven, didaktischen Ansätze nicht zu führen vermögen. Daher interessiert sich die aktuelle didaktische Debatte besonders für Kompetenzansätze. Dazu gehören die Provokation bereits vorhandener Kompetenzen und die Schulung zur Entfaltung und Förderung neuer Kompetenzen, was die vorliegende Studie durch den vorgeschlagenen Einsatz im LKU zu exerzieren versucht.

Landeskunde: Obwohl dem Begriff "LK" das Konturhafte fehlt, gilt sie als eines der ersten Fächer im DaF-Bereich, das die didaktische Bedeutung der Kompetenzförderung prinzipiell hervorhebt und sie als effizienteres Ziel des Faches als die Vermittlung von Faktenwissen betrachtet. Bei der Stoffauswahl handelt es sich um mögliche Angebote, einen landeskundlichen

العدد الرابع والعشرون (الجزء الثانى) 2018

()

مجلة كلية التربية- جامعة عين شمس41 
Kompetenzförderung im Landeskundeunterricht

Stoffkanon gibt es nicht. Kriterien der Auswahl wie die Berücksichtigung der institutionellen Rahmenbedingungen, der Zielgruppenkompetenz, Transferierbarkeit und der interkulturellen Bezüge der Texte sollen beachtet werden.

Der LK im Lehrplan der Deutschabteilung der pädagogischen Fakultät der Universität Ain Shams fehlt ein klar didaktisches Konzept. Die Wahlfächer sind problematisch, da die Entscheidung dafür nur der Lehrperson überlassen ist.

Kompetenzbildung: Die vorliegende Studie entwirft ein didaktisches Modell zum Einsatz der LK im FSU in Ägypten, das auf die sämtlichen kognitiven, kulturell-kommunikativen und interkulturellen Zielsetzungen der LK Rücksicht nimmt und dazu fächerübergreifend die Kompetenzen der Lernenden anspricht und weiterentwickelt.

LK-Ansatz: Durch eine Kette von durchdachten Aufgaben und den Aspekt von Unter-Druck-Setzen werden die Kompetenzen der Studierenden herausgefordert. Didaktisch gezielt werden erforderliche Schlüsselkompetenzen geschult und entfaltet. Die Versuchsgruppe hat nach 5 Wochen ihr Zeitmanagement durch Arbeitsteilung und Koordination optimiert, die die Entwicklung ihrer Teamwork- und Problemlösungskompetenz voraussetzt. In der 9. Woche zeigt sich eine tiefgreifende Entfaltung ihrer Kreativität-, Textsorten-, kommunikativen und interkulturellen Kompetenzen, indem der Prozess der Transformation der Lesetexte in Dialogszenen ganz souverän gelaufen ist. Perspektivenwechsel und -übernahme im Gespräch weisen eine zufriedenstellende Entwicklung der interkulturellen Kompetenz nach. Besonders in einer improvisierten Gesprächssequenz zeigt sich die Kompetenzentwicklung der Studierenden, die imstande geworden sind, kommunikativ angemessen $\mathrm{zu}$ handeln und plötzliche Probleme im Team zu bewältigen. 


\section{Literaturverzeichnis}

ABCD-Thesen zur Rolle der Landeskunde im Deutschunterricht. In: Deutsch als Fremdsprache. Leipzig, Heft 5/1990. 306-308.

BECK, Herbert 1993: Schlüsselqualifikationen. Bildung im Wandel. Darmstadt: Winkler Verlag.

BEER, Rudolf 2007: Bildungsstandards - Einstellungen von Lehrerinnen und Lehrern. In: Schulpädagogik und pädagogische Psychologie (Bd.1). Wien/Berlin/Münster: LIT-Verlag.

BISCHOF, Monika/Borchard, Beatrix 1987: Kontrastive Landeskunde. Ein Konzept für bi- und trinationale Seminare. München: GoetheInstitut-Manuskript.

BOLOGNA 1999:Der Europäische Hochschulraum. Gemeinsame Erklärung der Europäischen Bildungsminister 19. Juni 1999, Bologna. (Online-Dokument). Zugriff am 10.03.2010:https://www.bmbf.de/files/bologna_deu.pdf

BUTTJES, Dieter 1989: Landeskunde-Didaktik und landeskundliches Curriculum. In: Bausch/Christ/Hüllen/Krumm (Hrsg.): Handbuch Fremdsprachenunterricht. Tübingen: Francke Verlag. 112-119.

DRESSLER, Gunner et al 1980: Welche Landeskunde braucht der FU? Grundsätzliche Überlegungen zur Vermittlung von Sprache und Kultur im FU. In: Linguistik und Didaktik 11. 43/44. - München: Wilhelm Fink Verlag. 233-251.

DRÜGH, Heinz et al (Hrsg.) 2012: Germanistik. SprachwissenschaftLiteraturwissenschaft-Schlüssel-kompetenzen. Stuttgart/Weimar: Metzler.

DZYKOVYCH/Turysheva 2016: Die Überlegungen zur Auswahl des landeskundlichen Lehrstoffs. In: Вісник 8 / 2016. 39-44.

FLEMING, Clare 2015: Landeskunde Down Under? Ein Interview. In: Fremdsprache Deutsch. Landeskunde und kulturelles Lernen. Nr. 52. 2015. 55-57.

FRANK, Andrea/Haacke, Stefanie/Lahm, Swantje 2007: Schlüsselkompetenzen: Schreiben im Studium und Beruf. Stuttgart/Weimar: Metzler.

HERRDE, Dietrich./Marnette, Hua. et al.: Landeskunde. Überlegungen zu Theorie und Methode. Potsdam: (Hausdruck). Pädagogische Hochschule 1989.

العدد الرابع والعشرون (الجزء الثانى) 2018

مجلة كلية التربية- جامعة عين شمس 43 


\section{Kompetenzförderung im Landeskundeunterricht}

HILLER, Gundula Gwenn 2011: Schlüsselqualifikation Interkulturelle Kompetenz - Ein Bildungsauftrag der deutschen Hochschulen? In: Dreyer, Wilfried/ Hößler, Ulrich (Hrsg.): Perspektiven interkultureller Kompetenz. Göttingen: Vandenhoeck \& Ruprecht. 238-254.

HONOLKA, Harro (Hrsg.) 2003: Schlüsselqualifikationen. Das Plus eines universitären Studiums, Informationen für Studierende, Lehrende und Arbeitgeber. München: LMU München.

KRAMSCH, Claire 1991: Bausteine zu einer Kulturpädagogik des Fremdsprachenunterrichts. In: Jahrbuch Deutsch als Fremdsprache 17. München: iudicium. 104-120.

NEUNER, Gerhard 1994: Fremde Welt und eigene Erfahrung - Zum Wandel der Konzepte von Landeskunde für den fremdsprachlichen Deutschunterricht. In: Neuner, Gerhard (Hrsg.): Fremde Welt und eigene Wahrnehmung. Kassel: Universität Gesamtschule Kassel. 1439.

NÜNNING, Vera 2008: Schlüsselkompetenzen. Qualifikationen für Studium und Beruf. Stuttgart/Weimar: Metzler.

NÜNNING, Vera/Nünning, Ansgar 2000: Britisch cultural studies konkret. 10 Leitkonzepte für einen innovativen Kulturunterricht. In: Der fremdsprachliche Unterricht Englisch 1/2000. Seelze: Friedrich Verlag. 6-9.

OLECHOWSKI, Richard 1997: Schlussfolgerungen für eine Reform der Schulen der Vierzehn- bis Neunzehnjährigen unter dem Aspekt einer „humanen Schule“. In: Persy, Elisabeth/Tesar, Eva (Hrsg.): Die Zukunft der Schulen der Vierzehn- bis Neunzehnjährigen. Frankfurt am Main: Peter Lang. 361-376.

OTTEN, Matthias 2006: Profession und Kontext. Rahmenbedingungen der interkulturellen Kompetenzentwicklungen. In: Otten, Matthias/ Scheitza, Alexander/ Cnyrim, Andrea (Hrsg.): Interkulturelle Kompetenz im Wandel. Band 1: Grundlegungen, Konzepte und Diskurse. Frankfurt a. Main/London: IKO - Verlag für Interkulturelle Kommunikation. 57-90.

PAULDRACH, Andreas 1992: Eine unendliche Geschichte. Anmerkungen zur Situation der Landeskunde in den 90er Jahren. In: Fremdsprache Deutsch. Zeitschrift für die Praxis des Deutschunterrichts. München: Klett Verlag. Heft 6, 4-15. 


\section{Dr. Mahmoud Fouad}

PICHT, Robert 1980: Landeskunde und Textwissenschaft. In: Wierlacher, Alois (Hrsg.): Fremdsprache Deutsch. Bd. 1. München: W. Fink Verlag. 271-289.

RAASCH, Albert 1997: Der Fremdsprachenunterricht hat sich verändert - auch im Fach Französisch? In: Neusprachliche Mitteilungen aus Wissenschaft und Praxis. 2/1997. Berlin: Cornelsen. 68-74.

REDER, Anna 2011: Ausgewählte Fragen der Deutschdidaktik. unter:http://janus.ttk.pte.hu/tamop/tananyagok/dig_jegy_nemet/index .html (abgerufen am 12.02.2018)

RIEMER, Claudia 2007: Textrezeption und Textproduktion als fremd/zweitsprachliche Kompetenz. In: Bausch, Karl-Richard/Krumm, Hans-Jürgen et al. (Hrsg.): Textkompetenzen. Tübingen: Narr. 161166.

SCHMIDT, Siegfried J. 1980: Was ist bei der Selektion landeskundlichen Wissens zu berücksichtigen? In: Wierlacher, Alois (Hrsg.): Fremdsprache Deutsch. Bd. 1. München: W. Fink Verlag. 289-299.

STRAUB, Jürgen 2007: Kompetenz. In: Staub, Jürgen/Weidemann, Arne/Weideman, Doris (Hrsg.): Handbuch. Interkulturelle Kommunikation und Kompetenz. Grundbegriffe - Theorien Anwendungsfelder. Stuttgart: Metzler. 34-46.

TRIM, John/North, Brian/Coste, Daniel 2001: Gemeinsamer Europäischer Referenzrahmen für Sprachen. Sprachenlernen, lehren und beurteilen. Berlin/München: Langenscheidt.

WEINERT, Franz 2003: Leistungsmessungen in Schulen. Weinheim/Basel: Beltz.

WIERLACHER, Alois (Hrsg.) 1980: Fremdsprache Deutsch. Grundlagen und Verfahren der Germanistik als Fremdsprachenphilologie. 2 Bde. München: W. Fink Verlag. 
Kompetenzförderung im Landeskundeunterricht

\footnotetext{
العدد الرابع والعشرون (الجزء الثان) 2018 